\title{
Decision Making Processes in Online Social Networks: A Comparative Analysis of Health and Financial Online Social Networks
}

\author{
Valeria Sadovykh \\ PricewaterhouseCoopers and University of Auckland \\ valeria.a.sadovykh@sg.pwc.com
}

\author{
David Sundaram \\ University of Auckland \\ d.sundaram@auckland.ac.nz
}

\begin{abstract}
Health and finance are two aspects of life that are crucially important. Health and finance sites are the most frequently visited by online participants. The sheer volume of sites and the variety of information and advice available online on those two topics have left individuals with potentially greater support for their decision-making (DM) processes. This paper uses the Netnography qualitative research methodology to understand, explore, and identify the similarities and differences between, and unique features of Health and Financial Online Social Networks (OSNs). Specifically, this paper will study (1) the OSN - assisted DM process (2) the structure and sequence of DM phases in OSN's and (3) the behaviour of OSN participants across networks. The results suggest that the DM process is supported in both types of OSNs, but the structure and the sequence of DM phases, as well as the participation style across participants on these networks are different.
\end{abstract}

\section{Introduction and research motivation}

Financial OSN 1. Seeker 1: 2 year fixed interest at 6.5\%.... and with $100 \%$ protection. Too good to be true? If they say $100 \%$ guaranteed and quote a fixed interest rate - that's got to be true hasn't it? It's from and the above account is their 2 year growth and protect bond. Very tempting, true or false? www. co.uk/. 2. Adviser 1: Hi not sure about them, the company seems to be very new and the assets minimal. If I had money to invest I would want someone a bit more 'established www.companycheck/ 3 . Adviser 2: Have you checked whether they are registered members of the FCA? They are also a PLC so check on line to see the rules about that kind of company before you invest. ...

Health OSN 1. Seeker 1: Is there anyone out there that isn't getting immunizations for their baby? I have done a lot of research and I don't feel right about injecting my new-born with a bunch of $b / s$. Also does your doctor pressure you about the whole thing?

2. Adviser 1: I am a Medical Assistant and after going through my class there is no way I wouldn't want my son vaccinated. It is scary to know something foreign is being put into our babies but I feel that it would be a lot scarier for him to get so sick that his little body can't fight it off. I like the idea of one at a time to see reactions though You protecting your kids is also helping protect others as well. 3 . Adviser 2: I didnt vaccinate my son,i did alot of research on this...nd my heart said no to vaccines... His pediatrician said i should..
These two queries posted on two different types of OSNs share one main purpose: to gather enough information to make a life decision. Both decisions can lead to serious consequences based on the responses provided, whether in the financial example, investing money, or in the health example, the vaccination of a child. Both queries received a substantial number of responses and support for making a decision from random advisers who are not necessarily experts in the subject in question. Both threads provide some possible options to make a decision, but also could mislead the seekers on the potential future DM choice. It has been reported that $80 \%$ of adult Internet users use OSNs for general health information [11]. As for financial matters, it has been identified as one of the top ten aspects researched on the Internet [3]. OSNs became the most popular destination and potentially the first stop for Internet users in need of information to make a decision.

Sadovykh, et al. [24] define OSNs as online public / semi-public / private services / sites / platforms that facilitate the creation and reflection of social ties / networks / connections / relations among stakeholders / groups / organizations who share interests, activities, beliefs, dislikes, knowledge and / or values. OSNs are not only for generating connections and discussions between participants on the subject of interest, but also considered and used as a support tool for DM on various subjects of interest, as they provide their participants/stakeholders various forms of support, ranging from the instrumental to the emotional and informational [23, 24].

The influence of information systems and technology on the DM process cannot be overstated. Regardless of advances in related technology, when faced with a DM situation, it is reasonable to assume that the core processes are similar. The literature on decision-making is built on the existence of rational and sequential, as well as anarchical models and processes in traditional settings. However, given the social and behavioral nature of the DM process, it is necessary to find evidence in reality to confirm the 
existence of a common core, and specifically show what DM process is supported by OSNs. We suspect that the dynamics of DM may vary in different online social network environments that incorporate the participation of online 'advisors' and 'decisionmakers'. In order to establish the common core of the decision-making process in OSNs, a comparison of $\mathrm{DM}$ processes across different types of networks is required.

This study has been motivated and conducted based on prior research that specifically focused on HOSNs [23]. By analysing decision making process based on Simon's theory of rational decision making [26, 27] and interactions of HOSN's users, the authors questioned if the same decision-making behaviour, processes, structure and sequence will be reflected in different types of networks for different types of problems. This study not only looked at FOSNs, but also conducted a comparative analysis. The primary driver for this research is to study (1) the DM process supported in HOSNs and FOSNs, (2) the structure and the sequence of DM phases assisted by the use of HOSNs and FOSNs and (3) the behaviour patterns of decision- makers in both networks.

Health- and finance-related decision making are fundamental to most humans. There is an assumption that health and well-being decisions are more qualitative (subjective) and finance decisions are more quantitative (objective). Therefore, it is extremely important to understand how people make decisions in these different domains with different characteristics by using the emergent nature of online interactions and exchanges supported by digital platforms and infrastructures.

The results suggest that an OSN can, in fact, support and empower users in their DM (as can be observed in provided examples of OSN conversations provided). This was investigated by defining the OSNassisted DM process in both networks, so as to understand and establish whether that supposition is valid. However, the structure and the sequence of DM phases, as well as the participation styles of participants of those networks are different and this was observed through the reviewing and coding of participants' communications flows under the DM phases of the DM process developed by [27].

This paper is structured as follows: the next section will discuss seminal DM models, as well as newlydeveloped research artefacts for investigating online health and financial DM (Section 2). Thereafter, we discuss the chosen research methodology, Netnography, and why we consider it a good fit for this study (Section 3). This will result in a detailed description of the adapted netnographic research approach and how it has been applied (Sections 4 and
5). Section 5 also interprets the results of the comparative analysis of HOSNs and FOSNs. Section 6 concludes with discussion on research and practical implications.

\section{Decision making in HOSNs and FOSNs}

Orlovsky [22] defined DM as the act of a binary preference with a set of alternatives that are formulated and suggested to the decision-making person as their rational choices. According to [26], DM involves choosing issues that require attention, finding adequate courses of action, and selecting one alternative as the final decision. Simon [27] suggests that decision making is made up three phases namely intelligence, design and choice. Later, [13] extended this model by adding two other phases: implementation and monitoring. Cooke and Slack [7] developed the sequential model that uses Simon's model to explain decision making as a cyclical process that focuses around the problem; for them, problem solving is not merely the three phases of the Simon model, but a continuous process of identifying the best alternatives and courses of action. Mintzberg et al.'s [21] model follows the linear structure from Simon's rational DM process and reflects repetitive elements and incoherent phases of DM. In this model, the decision-maker comes with recognition of a problem or tangible request that requires an action, with the solution coming in a manner of different repetitive DM stages that do not necessarily follow the sequence.

Unlike rational and sequential models, DM theories have emerged into an anarchical problem-solving process that is driven by events. In other words, this model is a free decision-making process that is more intuitive than rational [17]. The decision-making process driven by events is similar to [6] the garbage can model of decision choice in which the four streams that interplay are problems, solutions, participants, and choice opportunities.

When we look into the online DM research, there are only few new concepts that have been proposed based on the existing foundational models. Sadovykh et al. [24] looked at Simon's DM process and tried to find evidence of its existence in OSNs. The findings did emphasise that the DM process is different in OSNs, but they can be utilized as a support tool for DM, especially as a provider of relevant information with only insubstantial investments required from participants.

Rarely are the risks associated with the use of OSNs foremost in our minds when we talk about OSNs for DM; for health, it is inaccurate self-diagnosing; in finance, there is a belief that those who advise on financial matters are also scammers; and, in general, 
information on OSNs is of poor quality with excessive influence from media, marketing and promotion; there is, too, always the question of the breach of privacy of decision-makers or anyone who participates in OSNs. Therefore, unsurprisingly, most researchers and practitioners underestimate the benefits and overestimate the risks of the available online resources [9]. Ferguson et al. [11] observe that the public is distracted by focusing on the negative aspects of OSNs and overlook their benefits. Although there are studies that illustrate and measure the amount of inaccurate information on health and finance networks, others show how OSNs are beneficial to users by providing emotional support and a comforting environment for communication and learning [9]. In the case of HOSNs it has been found that their role is not only to provide information but also to support stakeholders in their health DM [23]. Other studies found that HOSN users found them more helpful than offline social groups, and frequently more cooperative than physicians [5].

The area of trust in OSNs for health and finance is a well-researched one. For example, [12] studied how a decision-maker's sense of belonging to an online community directly correlates with emotional trust and positively affects the purchasing decision. Sillence et al. [25] came up with a framework for understanding trust factors in web-based health advice divided into three dimensions: heuristic analysis that refers to the look and the feel of OSNs; systematic evaluation of site content, which refers to the quality of the content and the participants of OSNs; and longer-term engagement through source integration and selfdisclosure processes which refers to the community quality, trust between the participants, and personalization. All of these factors were found to have an effect on trust in health OSNs.

Casarin et al [4] researched how the financial literacy of FOSN users affects the quality of DM and what is the presumption across financial advisers on the use of FOSNs for financial investment decisions. They found that knowledge sharing and learning in FOSNs cannot compensate for the financial education gap of the decision-makers and that the recent popularity of FOSNs tends to expose more risks in financial DM, therefore better financial DM is not guaranteed by the use of FOSNs. Only qualified financial literacy could promote the diversification of the risk in the making of FOSN-assisted decisions. Their findings doubt the concept of information quality, sharing and generation which are provided in FOSNs.

After reviewing the literature on HOSNs and FOSNs, it has been observed that academia focuses mainly on fragmented aspects of DM in OSNs: either the decision-maker in the process of decision making (cognitive process); alternatives and risks associated (courses of action or opinion); and decision (final choice) and factors contributing to it. But there is a scarcity of research that looks at it as an eco-system the decision-making eco-system; where the decisionmaker goes through the entirety of their decisionmaking process by using OSNs. This study tries to look into the whole picture of the DM process by use of OSN by using Netnography as the primary research method.

\section{Research methodology}

Health, finance, OSNs, and decision making have attracted much interest in academia. However, there is a paucity of research on a combination of these themes. Moreover, there is no evidence of any studies of decision-making differences in the areas of finance and health using OSNs. The nature of the research objectives and goals, and the domain of this research evidently demand an approach that can explore, understand, compare and contrast how HOSNs and FOSNs can support the DM process.

The goal of qualitative research is to achieve an understanding of human behaviour in a particular situation and the context within which it acts [14]. Such research is more suitable when there is a need to analyze a phenomenon that cannot be statistically proven and when the data cannot be converted to numbers. In this research it would be difficult to quantify the decision-making process and phases used in either HOSNs or FOSNs. Qualitative methods employ data in the form of words, such as transcripts of open-ended conversations or written observational descriptions of activities. Such data can be analyzed in ways that retain their inherent textual nature [14].

Many of us live our lives in an inter-related matrix of online and offline social behaviors. Every day, individuals connect to each other through online boards, blogs, wikis, web pages, list servers, multiusers, dungeons and chat rooms. Netnography is a new social science approach to the study of online communications through ethnographic research that combines participation and observation of online communications with new forms of digital and network data collection, analysis and research representation [16]. Netnography does not deal merely with words in online posts; it also considers images, audio, video, and other digital artifacts $[15,16]$. Based on our research objectives, Netnography seemed to be an appropriate methodology that can assist in understanding the behaviour of online users and their experience in OSNs, and evaluate the existence of DM processes, structure and phases. This research follows the Netnography method made up of planning and entrée 
(section 4), data selection, collection, and analysis (section 5) and discussion and findings (section 6).

\section{Planning and entrée}

The planning step is about defining the research objectives and questions. The entree involves the choice of networks of interest where observation takes place, and data collection can then proceed. Kozinets [15] recommends that conversation and topics should be popular with much interaction and many visitors.

This research combines two studies, the first conducted on HOSNs [23], where the 11 most popular health topics discussed on the Internet were selected [11], these topics being: Asthma, Alcohol and drug addiction, Bulimia, Cancer, Depression, General Health problems/medicine, HIV/AIDS, Plastic Surgery, Pregnancy, Scoliosis Problem, and Weight Loss/Appearance issues. The second study was on FOSNs, where the categorization of online networks provided by [18] was adopted and modified. The three FOSN categories in this study are: retail, support services and professional. Retail (Consumer) financial service providers have a strong sense of connection between participants on common subjects of interest, such as credit card, mortgage or insurance service offerings. Support Services are designed for those seeking support or answers on a finance-related matter, like advice on budgeting or on share, bond and technology prices. Professional (Wholesale) networks are where professionals meet for financial transactions. There is an expectation that FOSN users will have some level of knowledge and expertise in financerelated matters for this type of networks.

It is important to ensure that the chosen sites for studies cover most of stakeholders; in the case of health we tried to capture conversations with contributions from health experts, caregivers, patients and doctors; and for finance, professionals employed by financial institutions, and financial experts; and, for both topics, random users interacting, posing questions and offering opinions, not necessarily simultaneously nor on one network.

Another important step in the initial phase of netnography is an understanding of the participants on selected networks. The researchers have classified online participants along lines similar to that of Kozinets typology - Tourists, Minglers, Devotees, and Insiders [19]. We classified the participants as Advisers, Seekers, Experts, and Observers. Advisers provide support to seekers in order to solve a problem. Advisers either can support decision-makers or mislead them. Seekers are interested in immediate results advice provided by advisers. Once their needs are satisfied, their relationships with the community might dissolve. Experts have strong ties within the community and their respect mainly depends on their profile, on which can be displayed their expertise, education, and volume and past history of participation. Sometimes experts appear as moderators of OSN. Observers are silent members whose numbers cannot be easily established.

\section{Data selection, collection, and analysis}

The second step in the netnographic research framework involves data selection and collection, a delicate and important procedure that serves many purposes in the research approach [1]. Kozinets [15, 16] recommends obtaining three different types of data during the collection process: archival data, elicited data, and field notes. This study took two types of data for the collection process: first, the written communications between different stakeholders that occurred in the online communities (archival data); and second, the researcher's self-authored field notes, in which the observation ideas and comments were recorded and synthesized in the analysis section. Obtaining field notes for each conversation and the communities observed allows the first fresh research perspectives on the data collected to be captured without a delay. Those derived from the observational process were mainly about the participants' behaviour, OSN design, conversation styles for each community, codification of different phases and difference, financial and health tools offered to users, and comparing/contrasting the networks.

To find suitable posts, the researchers utilised search engines like Google and Yahoo Groups by keying keywords such as: top health \& finance forums, top health \& finance online advisers, and best health \& finance virtual communities \& forums. After identifying and selecting networks of interest, the researchers tried to become familiar with the network culture by reading terms and conditions; searching for popular topics, and topics by last date modified; checking moderators profiles and their posts; and understanding any other website design components that could influence the immediate attitude towards the network and subsequently affect the decision-making process. Only English-language websites were chosen for analysis. Furthermore, most were hosted in the US although users were distributed all over the world. The first study was conducted on HOSNs and took place in 2013; the FOSN study took place in late 2015 and was completed by mid-2016, taking into consideration lessons learned from the HOSN study.

For the health study, we chose 29 websites and selected 51 conversations associated with the DM processes, phases, and problem solving themes. For the 
finance study, we chose 21 websites from which 50 conversations were collected following the same selection criteria as for the health study. The chosen conversations for the two networks were on the top topics of interest identified by the participants from each study domain. For both studies, this data collection process ensured that the conversations were not on a single subject and not conducted by the same participants; therefore the DM process is not repetitive, and different stakeholders represent a range of users and the voice of different demographics. The effects of bandwagon and confirmation biases of information processing were alleviated by choosing three to four different topics under each health and finance category. All conversations were collected and separated according to the subject of interest as per HOSNs and FOSNs and phases of decision making (as per Simon's model). Each post was assigned a specific code that indicates the post subject correlated with the subject of interest. Each has statistical information regarding the numbers of conversations, words and participants.

The data collection process is a major challenge, especially when using the Internet as the main source; there is always a danger of getting lost with an excessive amount of data and field notes [15], [19]. There is always an assumption that there are more data out there that can bring more insights. The solution to this is in research planning, closely following the research process, absorption of research objectives and questions only. Once conversations, posts and websites that are directly related to the research objectives are identified and the data collection process has taken place, data-sorting begins. For the latter, considered to be at the heart of any research study [8], in addition to [15] advice to use content and discourse analysis, a third component, conversational analysis, was added. The rationale to add another component was to tailor the analysis to the nature of the research objectives: to compare and contrast conversations around decision making within different networks.

Content analysis is used to expedite the coding procedure and analysis of data. Discourse analysis is a general term for written, spoken or signed language analysis. Conversational analysis is important for research that tries to understand the context, meanings and trends of conversations and communities of interest. All three analyzes do have similarities and differences, which is why, in combination, they are able to produce a better quality research analysis.

Coding and Noting, the second step in the analysis process, adds additional classification to the collected data, as it helps the data to be an organized sequence and pattern based on research objectives rather than disparate bits of information. The conversation patterns were categorized in accordance with the phases of
Simon's DM model [27]. In the repetitive process of analysis, with additional exploration of data, literature and pre-formed findings, the additional phases of the DM process were established, as were new categories, based on the existing phases of Simon's DM model [27], for further analysis and coding. The final step was the comparing and contrasting of DM phases, structure, sequence, and participant behaviors in HOSNs and FOSNs. The analysis findings will be further discussed in the next section.

\section{Findings and discussion}

The data analysis process followed the netnography and grounded theory guidelines, but the content, conversation and discourse analysis added imperative details, quantitative view and richness to the entire research process and findings $[15,16]$.

Content analysis has been used in this netnographic study to identify the weightings of the five phases of the DM process, and to understand who mainly generated and provided content. The text has been broken down by countable units such as phases and categories of the DM process by Simon [26, 27]. The analysis indicated that there are more advisers in OSNs than seekers. In both networks it has been assumed that advisers are willing to share either finance- or health- related information or knowledge with other participants.

As for DM phases, the design phases of advisers have the highest score, meaning that advisers propose the models for decision making that consist of alternatives and options that can accommodate the seeker's query. The actual number of implementation phases of advisers shows how many times they shared their decision experience with the HOSN and FOSN public. For FOSNs, there is a good pattern of choice phase from the seeker perspective: some returned to the network to acknowledge that the choice had been made (as per the prologue example). Thus, the conversations on FOSN do not necessarily reach every phase of the DM process. This entails the question of the behaviour of participants, their participation style and DM style. For health, this is different: the choice, implementation and monitoring phase were rarely seen in the conversations streams, and in most cases seekers would not come back and share their experience and the consequences of the decision made.

As for the nature of the content, what was insightful is that health conversations retained their relevance over time, and the timing of the query posted online is irrelevant as health concerns are recurrent; for example, a diabetic post five years ago would still be relevant. In finance, timing of query did matter in most of the FOSN's categories and conversations; for 
example, conversations on oil prices five years apart would be very different. Therefore, in finance, only rarely would a conversation post be active long after the initial post.

As for information quality and content provided, it was found that financial information was more scientifically structured than that provided in HOSNs. The information or advice provided in health might not always sound logical, but because HOSNs had a tendency to be more emotional, it can be assumed that the participants accepted information (advice) that was, for example, not necessarily based on specific medical knowledge (non-traditional medicine, detoxing and fasting, spiritual healing for cancer patients, etc.).

Conversational analysis was used in this study to describe the orderliness, structure and sequential pattern of participants' interaction in HOSNs and FOSNs. As previously described, the conversations were broken down into phases of the DM process and during the analysis process new steps and categories were identified. New steps such as 'entry', 'exit' and 'background information' showed that conversations in HOSNs were following a real - life structure, where participants introduce themselves by providing background information and exit the conversation when required. On FOSNs, the new conversational pattern that established its presence is 'share of previous experience', 'personal opinion', and 'confirmation of someone's choice, opinion or advice'. Table 1 below summarizes the above and shows comparisons between HOSNs and FOSNs.

Figure 1 shows examples of conversations that have been analysed and the new steps discovered for each conversation. Conversational analysis also revealed the phenomenon of adviser experience in financial networks: the adviser, when describing his decision experience, tended to follow the sequence of phases of the DM process but backwards. Nearly all phases of the DM process have been attended by FOSN users at least once from each post; this could not be observed on HOSNs; the choice, implementation, and monitoring have the least attendance by HOSNs users. This could be explained by advisers not wanting to admit responsibility for the consequences of a decision taken by someone who followed their advice. In contrast, health networks offered strong emotional support and some sort of common bond between participants, which was not observed in finance, even when the matter was serious and assumed some emotional support was required (e.g. loss of family savings in the stock market).

Discourse analysis helped to understand the underlying mood of conversations and behavioral aspects of OSN users, the context behind the texts. It has been identified that there are many conversations that contained advertisements rather than actual advice on a problem/decision. In finance, advertising is explicit, being the way professional financial advisers make money, and therefore they are available at any time to give a clear option/advice for a seeker's question/query. As for health, advertising was mainly hidden; usually people would advise on medicine, and/or provide the link to buy the product online and assure everyone that the product suggested is working in their case. This appeared especially in conversations about weight loss.

\subsection{Structure and sequence}

To understand the structure and sequence of HOSNs and FOSNs and evolved decision-making process across participants, this study coded the collected conversations to Simon's DM-process phases [26, 27]. Intelligence Phase (I): when a decision-maker is capable of retrieving information in real time in seconds. OSNs are not standard search engines, but can provide information according to the search query or problem. It was evident that, through the use of OSNs, decision-makers can find similar problems and alreadydeveloped solutions that have been tested and evaluated by other members of networks. Therefore, it can be expected that both types of networks can enhance the intelligence phase of the decision-making process by providing access to a variety of data sources and formats (visual, textual, mathematical, and graphical). Design Phase (D): is all about alternatives and models of outcomes and consequences and additional questions that might lead to a better design option for DM. OSNs provide an opportunity for decision-makers to explore alternatives by simply asking for advice or browsing through the different threads and posts of interest, whether health - or finance-related. In both cases, this phase can be attenuated by simply presenting alreadydeveloped models of solutions that were provided by other members of the OSNs. This was evident in financial networks, for example where participants sometimes provided logical models on how and where to invest money and what the expected return might be.

The decision-makers of OSNs are not required to accept the provided models, but they can evaluate them and find them useful or otherwise. Choice Phase (C): was to be found in FOSNs, specifically in professional networks where investors could replicate the adviser's strategy and show their financial gains or losses; it could also be seen in the posts where a seeker returns to the thread to post the choice made or acknowledge that the thread had been reviewed and used in a reallife environment. As for health, the choice made by a seeker in most cases was not transparent in the observed text conversations. Implementation and 
Monitoring (IM and M): The implementation phase was found to be present in FOSNs, even though originally it was anticipated that it would be difficult to observe. Monitoring could be detected in financial professional or retail networks, mainly when seekers were returning to share the results and consequences of their decisions. As for health, a seeker implementation phase was not always apparent and could only be observed if a seeker returned to the community and confirmed the implemented choice in real life. This can be explained by the fact that implementation is an actual 'doing' process that does not involve textual presentation (texts are our thoughts) and that actions are more about physical processes. Especially in health, the implementation phase can take longer to show the consequence of a decision.

Figure 1 below displays recorded examples of analysed conversations for each of the studied networks. RT03 means that the post falls within the Retail (Consumer) category and is numbered 03 and the heading is 'Funds vs. Direct' which implies that a decision-maker asked for opinions on whether to invest in products (funds) or just buy stocks and bonds. The second example is under the category of General Health and numbered 01, titled: 'Hyperthyroidism and bowel problems'.

The diagrams show how the phases of the DM process are interconnected in the online environment that causes it to follow an anarchical structure. The phases of the DM process are visible, but the sequence in which the conversations move between them is unstructured and appears random, both for health and finance. As discussed before, the phases of choice, implementation and monitoring are difficult to capture and in the case of GH-01, these phases are absent, unlike in finance conversations.

A striking finding in FOSNs is the difference between how advisers post their choices made in the past as part of their previous experience and how seekers provide background information based on their experiences of past decisions. Advisers, when suggesting a choice, usually start the conversation with a clear statement - the choice to be made - and then proceed with a description of their advice and reasoning (Design-Model (D) - Intelligence (I)). Seekers usually follow the opposite sequence when explaining their DM, starting with Intelligence (I) background information on the decision to be (already) made; Choice (C) and/or need; and Design-Model (D) - options and alternatives available to them. In conversations about health, the structure cannot be clearly seen because of the high levels of emotions or unnecessary information that cannot be categorized under any DM phase.

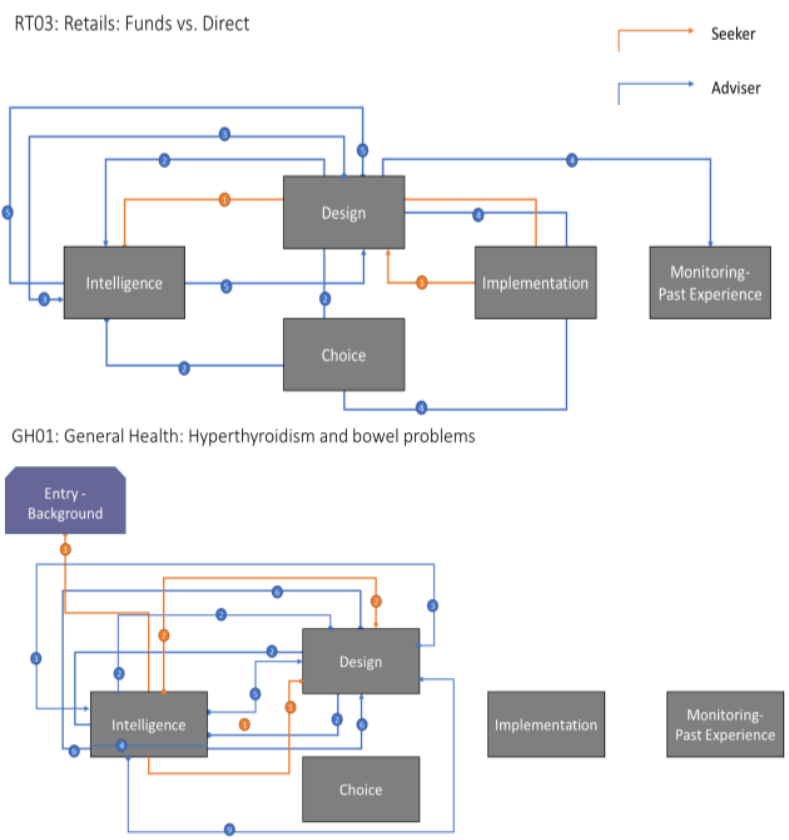

Figure 1. Analysis of DM phases, structure and sequence in FOSN and HOSN

To conclude the discussion on the structure and sequence for health and finance networks, the study can confirm that there is a structure in FOSNs and it is more widely present than in HOSNs. Also, this structure does not follow Simon's model; for finance it is more information-orientated and advice is given on facts and experiences shared. With HOSNs, participants are more emotional and advice is given freely but without much logical or scientifically proven back up. As for sequence, neither network follows Simon's sequence or rational human behaviour. The sequence of DM phases tends to be more anarchical and sequential, following the Mintzberg model [1], factoring in the various dynamics of the DM process and a decison-maker.

\subsection{Behavioral patterns of participants}

The reason why this study is concerned with the behavioral patterns of participants is to understand how OSNs change the practice of making decisions and how that correlates with the theory of the rational decision maker. These research objectives translate into identification of those behavioral patterns that are: (a) are the same and/or different across two OSNs; (b) are new in either HOSNs or FOSNs and identical to each other; (c) have no presence in either.

The behavioral patterns even differed in FOSNs across the three categories. In the support services of FOSNs, advisers provided detailed solutions to the financial problems of seekers; much of the advisers' attention was on the design phase, presenting models, 
alternatives and options for a solution: a significant amount of discussion covered the execution process of the choice phase. In the retail services of FOSNs, participants were less engaged in explaining how to execute a provided 'choice', as it is assumed that participants are well acquainted with finance and quite aware of their actions and consequences. Also, retail services had more professional users, where it can be assumed that people have little time to over-explain their advice or provide guidance on how to execute their advice. Most conversations in retail services were logical, evidential, structured, and unemotional. The retail service is also about self-advertising and some people using FOSNs to profit financially from their advice or find potential customers. These networks mostly have a community of experts and even by observing conversations, users might benefit by gaining knowledge. Seekers in these networks feel obligated to return to acknowledge their choice and provide feedback, so therefore the implementation phase in this category of FOSNs showed its existence in comparison to the support services category. The behaviour patterns of the users of professional services are even more structured than in retail services; conversations conducted on those networks are much shorter and usually within the three phases of DM process, having on average only two lines of text. The purpose of interaction on these networks for FOSN users is purely materialistic: no one wants to waste time, it is all about past, present and future transactions. Users on professional networks tend not to share much of their previous experience; they mainly shared valuable information on future decisions or on the execution of these decisions. It can be assumed that users wanted to share neither their financial success stories nor failures. On such networks, users are accustomed to using financial acronyms and jargon.

In HOSNs, it was evident that participants are more willing to share their personal life, spending time on the creation of their personal profiles, and sharing their background and health history (cancer stage, personal and intimate relationship struggles, etc.). In the beauty and plastic surgery networks, people were not embarrassed to share their photos and ask for people's opinions on their look and what plastic surgeries to undertake to improve their appearance.

One perplexing observation is that, grammatically, financial conversations made more sense and sentences were more structured. Even in the example in the prologue it can be seen that users of health networks are less concerned with grammatical accuracy, using shortcuts. For FOSNs, the main challenge was the use of abbreviations that most non-insiders would consider jargon. The gender aspect also differed across the two networks: it is assumed there are more female participants in OSNs [12]. For HOSNs this general observation can be confirmed, but male participants predominate on professional and retail networks in FOSNs. Another surprising observation was that in FOSNs, seekers and advisers had a tendency to come back to conversations and post their decisions or follow the conversation thread and make more than one comment or provide advice. This behaviour was not observed in HOSNs; seekers tended not to return and advisers, after providing advice, tended not to follow up. In health, neither the location of the participant nor the date of the post plays a significant role. The advisers in health are predominantly those who experienced a similar concern and wish to advise; in finance this is not necessarily the case, as participants base their advice on their knowledge, expertise and available information (resources), while sometimes it is country- (or region-) specific. The identified behavioral patterns of online participants could, at a later stage, be translated into guidelines for better utilization of OSNs as a tool for decision making.

\subsection{Compare and contrast}

In this study we have attempted to find patterns of similarity and differences within the decision-making process and phases, and participants' behavioural patterns between HOSNs and FOSNs. Once the study on HOSNs was completed and the study on FOSNs began, the researchers kept track of all the differences and similarities in the field notes. The summary of the findings is presented in Table 1 .

\section{Conclusion}

The primary objectives of this study were to understand where there are similarities and differences, and what is unique in the way decisions are structured and sequenced, and how behaviour is different in these two networks. The findings suggest that the DM is supported by use of OSNs, but the structure and the sequence of DM phases, as well as the participation style and behavior across participants of those networks, are different. In observed conversations the DM phases are interconnected in various sequences. However, there is no one pattern that can reveal the cognitive process of decision-makers, and the way they use these phases in OSNs for DM is different. The orders of the DM phases tend to be more anarchical and sequential, following the Mintzberg model [1], [21], factoring in the various dynamics of the DM process and a decision-maker. These results challenge purely rational and anarchical models by recognizing 
the interweaving of anarchical decision sequences

within the structure of rational decision-making phases.

Table 1. Compare and contrast analysis between Health and Financial OSNs

\begin{tabular}{|c|c|c|c|}
\hline & Health OSNs & Financial OSNs \\
\hline \multirow{6}{*}{ 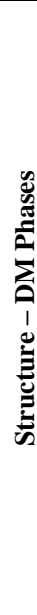 } & Intelligence & $\begin{array}{l}\text { Seeker: Supported; Adviser: Supported } \\
\text { Participants spend lots of time at the intelligence phase, } \\
\text { providing the background and description of the problem }\end{array}$ & $\begin{array}{l}\text { Seeker: Supported; Adviser: Supported; Participants do } \\
\text { not spend much time going into the background of their } \\
\text { query; this is a quick phase and a quick response with } \\
\text { alternatives and options expected }\end{array}$ \\
\hline & Design & $\begin{array}{l}\text { Seeker: Supported; Adviser: Supported } \\
\text { Most advices provided are based on the participant's } \\
\text { instinctive feeling or previous experience, sometimes } \\
\text { personal opinions. }\end{array}$ & $\begin{array}{l}\text { Seeker: Supported; Adviser: Supported; The design } \\
\text { phase is heavily utilized by participants who use it for the } \\
\text { strategies and options description based on facts, } \\
\text { knowledge, experience and logic }\end{array}$ \\
\hline & Choice & $\begin{array}{l}\text { Seeker; Not Supported; Adviser: Supported through } \\
\text { sharing previous experience; Choice is not visible in most } \\
\text { conversations; Choice advice can be provided based on } \\
\text { past experience only. }\end{array}$ & $\begin{array}{l}\text { Seeker: Partially - Supported based on the thread and } \\
\text { network category; Adviser: Supported through sharing } \\
\text { previous experience; Choice can be found from the previous } \\
\text { experience of advisers \& seekers }\end{array}$ \\
\hline & Implement & $\begin{array}{l}\text { Seeker: Not Supported; Adviser: Partially- Supported } \\
\text { if implementation refers to previous experience. }\end{array}$ & $\begin{array}{l}\text { Seeker: Supported- based on the thread and network } \\
\text { category; Adviser: Supported through sharing experience }\end{array}$ \\
\hline & Monitor & $\begin{array}{l}\text { Seeker: Not Supported ; Adviser: Partially-Supported } \\
\text { if Adviser refers to experience; in medical description of } \\
\text { side effects or any post implementation feeling }\end{array}$ & $\begin{array}{l}\text { Seeker: Semi-Supported- based on the thread, } \\
\text { Adviser: Supported through Adviser sharing experience; } \\
\text { Monitoring can be observed; Most of the time quantifiable }\end{array}$ \\
\hline & $\begin{array}{l}\text { New } \\
\text { Phases }\end{array}$ & $\begin{array}{l}\text { Entry, Exit, Background, Emotional Support, Previous } \\
\text { Experience }\end{array}$ & $\begin{array}{l}\text { Previous Experience, Personal Opinion, Confirmation of } \\
\text { Choice }\end{array}$ \\
\hline \multicolumn{2}{|c|}{$\begin{array}{l}\text { Sequence - } \\
\text { DM Process }\end{array}$} & $\begin{array}{l}\text { Intelligence to Design (I-D) sequence of phases is present } \\
\text { in most conversations; }\end{array}$ & $\begin{array}{l}\text { Design to Intelligence (D-I), Choice to Intelligence (C-I) } \\
\text { are the most common patterns of conversations; the } \\
\text { sequence of phases is backwards: Design to Intelligence }\end{array}$ \\
\hline \multicolumn{2}{|c|}{$\begin{array}{l}\text { Participants } \\
\text { Behaviour }\end{array}$} & $\begin{array}{l}\text { Seekers rarely make reappearance on the posted threads; } \\
\text { Participants can become emotional and express strong } \\
\text { emotions, strong opinions (e.g. abortion, vaccination). }\end{array}$ & $\begin{array}{l}\text { Seekers have a tendency to come back to posted threads; } \\
\text { Advisers' past experience based on previous knowledge and } \\
\text { backed up with logical models; no strong opinions or debates } \\
\text { present on the networks }\end{array}$ \\
\hline \multicolumn{2}{|c|}{$\begin{array}{l}\text { Network } \\
\text { Categories }\end{array}$} & $\begin{array}{l}\text { Generic networks that cover a range of health categories } \\
\text { or specialized health networks; Professional networks for } \\
\text { doctors and nurses are not available for public. }\end{array}$ & $\begin{array}{l}\text { Ranges from professional, personal, support services, } \\
\text { commercialized, educational, and regulatory. }\end{array}$ \\
\hline \multicolumn{2}{|c|}{ Advertising } & Hidden & Explicit \\
\hline \multicolumn{2}{|c|}{$\begin{array}{l}\text { Trust \& } \\
\text { Quality }\end{array}$} & $\begin{array}{l}\text { Anonymity due to sensitivity of topic - strict sign-up rules, } \\
\text { but within the conversations participants collaborate closely } \\
\text { and share their lives }\end{array}$ & $\begin{array}{l}\text { Anonymity is not relevant; trust and quality of conversations } \\
\text { depends on network category and financial literacy of } \\
\text { participants. }\end{array}$ \\
\hline \multicolumn{2}{|c|}{$\begin{array}{l}\text { Networks } \\
\text { Design }\end{array}$} & $\begin{array}{l}\text { Modern website designs, with features to enhance } \\
\text { communication (icons, account statistics and profiles) }\end{array}$ & $\begin{array}{l}\text { Simple website layouts and categorization of topics, without } \\
\text { an emphasis on design features. }\end{array}$ \\
\hline \multicolumn{2}{|c|}{ Other } & $\begin{array}{l}\text { Location and timing irrelevant for support for DM, as } \\
\text { health conditions are not dependent on these factors. }\end{array}$ & $\begin{array}{l}\text { Location and timing are very relevant (i.e. regulations on } \\
\text { investments, changes in market and share prices over time) }\end{array}$ \\
\hline
\end{tabular}

This study revealed that the traditional decisionmaking process, models and theories that have underpinned the design and implementation of decision support systems for the past five decades are no longer applicable to the current environment of online interaction. The research shows that most of the decision-making phases identified by Simon $[26,27]$ and Mintzberg [1], [21] are present in HOSNs and FOSNs, but the sequence of these phases tend to be neither purely anarchical nor rational but a blend of both. The findings of the research have significant practical implications for the design of OSNs that support blended decision-making processes by leveraging the wisdom of crowds. We suggest that HOSNs, FOSNs and other OSNs need to differentiate themselves and provide appropriate mechanisms to attract, sustain and support their participants.

The difference between real and online worlds is how people present and describe their future decisions or the experience of DM processes they have already had. OSNs can help the decision-maker in identifying and providing the tools and resources that can enhance the DM process. Moreover, OSNs can assist users in conducting a post-analysis evaluation of their decision and address the potential use of OSNs as a support tool for the process of making decisions about health and finance. Economic theory states that when the individual is faced with a situation where a decision has to be made, the decision-maker experiences scarcity of resources and support for the choice determination [2], [26, 27]. OSNs are one of the providers of additional resources, or, in other words, another information channel that helps to determine the choice options [20].

Online communities are real, significant and growing. Organizations have only started to scratch the surface of how technology can help to build these communities. Individuals also only started to discover 
the whole potential and abilities of OSNs for DM. It is not the technological capability that is important; it is the ability of new technology ideas to capture communities' trust, i.e. managing risk and reward. What is needed is an understanding for building OSNs that will succeed if they attract people, engage people, retain people, build trust and spread to new people.

\section{References}

[1] Ahmed, A., Bwisa, H., Otieno, R, and Karanja, K, "Strategic Decision Making: Process, Models, and Theories", Business Management and Strategy, 2014, 5(1), p.78.

[2] Buchanan, L, and O'Connell, A, "A brief history of decision-making", Harvard Business Review, 2006, 84(1), $32-41$.

[3] Campbell, J, Investor Empowerment or Market Manipulation in Financial Virtual Communities, In S. Dasgupta (Ed.), Encyclopedia of Virtual Communities and Technologies, 2006, pp. 296-301.

[4] Casarin, R., Casnici, N., Dondio, P, and Squazzoni, F, "Back to Basics! The Educational Gap of Online Investors and the Conundrum of Virtual Communities", Journal of Financial Management, Markets and Institutions, 2015, 3(1), pp. 51-69.

[5] Cline, R. J. W, and Haynes, K. M, "Consumer health information seeking on the Internet: the state of the art", Health Education Research, 2001, 16(6), pp. 671-692.

[6] Cohen, M. D. and March, J. G, "A Garbage Can Model of Organizational Choice", Administrative Science Quarterly, 1972, 17(1), pp.1-25.

[7] Cooke, S., and Slack, N, Making Management Decisions, Great Britain, Hall Europe, 1991.

[8] Dubé, L, and Paré, G, "Rigor in Information Systems Positivist Case Research: Current Practices, Trends, and Recommendations", MIS Quarterly, 2003, 27(4), pp. 597636.

[9] Eysenbach, G., Powell, J., Englesakis, M., Rizo, C., and Stern, A, "Health related virtual communities and electronic support groups: systematic review of the effects of online peer to peer interactions", BMJ, 2004, 328(7449), p.1166.

[10] Ferguson, T, and Frydman, G, "The first generation of epatients”, BMJ, 2004, 328(7449), pp.1148-1149.

[11] Fox, S, and Brenner, J, "Family Caregivers Online", Pew Internet: a project of the Pew Research Center, 2012.

[12] Huang, L.T, and Farn, C.K, "Effects of virtual communities on purchasing decision-making: The moderating role of information activities", PACIS Proceedings, 2009, p 55.
[13] Huber, G. P, and McDaniel, R. R, "The DecisionMaking Paradigm of Organizational Design", Management Science, 1986, 32(5), pp.572-589.

[14] Kaplan, B, and Maxwell, J, Qualitative Research Methods for Evaluating Computer Information Systems, In J. Anderson \& C. Aydin (Eds.), Evaluating the Organizational Impact of Healthcare Information Systems, New York, Springer, 2005

[15] Kozinets, R. V, Netnography: Doing Ethnographic Research Online: Sage Publications, 2010.

[16] Kozinets, R.V, Netnography: Redefined, Sage, 2015.

[17] Langley, A., Mintzberg, H., Pitcher, P., Posada, E., and Saint-Macary, J, "Opening up decision-making: The View from the Black Stool", Organisation Science, 1995, 6(3), pp. 260-279.

[18] Mainelli, M, "Risk/reward in virtual financial communities. Information Services and Use”, 2003, 23(1), pp. 9-17.

[19] Malterud, K, "Qualitative research: standards, challenges, and guidelines”, The Lancet, 2001, 358(9280), pp. 483-488.

[20] Mayer, A, "Online social networks in economics", Decision Support Systems, 2009, 47(3), pp. 169-184.

[21] Mintzberg, H., Raisinghani, D., and Théorêt, A, "The Structure of "Unstructured"', Decision Processes. Administrative Science Quarterly, 1976, 21(2), 246-275.

[22] Orlovsky, S. A, "Decision-making with a fuzzy preference relation", Fuzzy Sets and Systems, 1978, 1(3), pp.155-167

[23] Sadovykh, V., Sundaram, D, and Piramuthu, S, "Do decision-making structure and sequence exist in health online social networks?", Decision Support Systems, 2015, 74, pp. 102-120.

[24] Sadovykh, V., Sundaram, D, and Piramuthu, S, "Do online social networks support decision-making?", Decision Support Systems, 2015,70, pp.15-30.

[25] Sillence, E., Briggs, P., Harris, P, and Fishwick, L., “A framework for understanding trust factors in web-based health advice", International Journal of Human-Computer Studies, 2006, 64(8), pp. 697-713.

[26] Simon, H. A, "Decision-making and Problem Solving", report of the Research Briefing Panel on Decision-making and Problem Solving, Washington, DC: National Academy of Sciences, 1986.

[27] Simon, H. A, "Theories of Decision Making in Economics and Behavioral Science", The American Economic Review, 1959, 49(3), pp.253-283. 